\title{
Characteristics of Adduct Spasmodic Dysphonia and Muscle Tension Dysphonia Using Spectrogram
}

\author{
Hyun-Hwa Lee ${ }^{1}$, So-Yoon Lee ${ }^{2}$, and Young-Hak Park ${ }^{1}$ \\ ${ }^{1}$ Department of Otorhinolaryngology-Head and Neck Surgery, Yeouido St. Mary's Hospital, College of Medicine, \\ The Catholic University of Korea, Seoul; and ${ }^{2}$ Department of Otorhinolaryngology-Head and Neck Surgery, Bundang CHA Hospital, \\ College of Medicine, CHA University, Seongnam, Korea
}

\section{스펙트로그램을 통한 내전형 연축성 발성장애와 근긴장성 발성장애의 특성연구}

이현화 ${ }^{1} \cdot$ 이소윤 ${ }^{2} \cdot$ 박영학 $^{1}$

가톨릭대학교 의과대학 여의도성모병원 이비인후과학교실, ${ }^{1} \mathrm{CHA}$ 의과학대학교 분당차병원 이비인후-두경부외과 ${ }^{2}$

\author{
Received January 12,2015 \\ Revised April 1,2015 \\ Accepted April 28, 2015 \\ Address for correspondence \\ Young-Hak Park, MD \\ Department of Otorhinolaryngology- \\ Head and Neck Surgery, \\ Yeouido St. Mary's Hospital, \\ College of Medicine, \\ The Catholic University of Korea, \\ 10 63-ro, Yeongdeungpo-gu, Seoul \\ 150-713, Korea \\ Tel $+82-2-3779-1054$ \\ Fax $+82-2-786-1108$ \\ E-mail yhpark7@catholic.ac.kr
}

Background and Objectives Adduct spasmodic dysphonia (ADSD) is a neurogenic and focal laryngeal dystonia resulting in a strained voice quality with spastic voice breaks. While muscle tension dysphonia (MTD) is caused by functional voice disorders, its symptoms are similar to those of ADSD. Because the approaches of treatment for ADSD and MTD are radically different, accurate evaluations are necessary for precise diagnosis. A spectrogram analysis for differentiating ADSD from MTD provides differentiations on four spectral findings (abrupt voice breaks, irregular wide-spaced vertical striations, well-defined formants and high-frequency spectral noise). The aim of this study was to evaluate if the spectrogram could provide detailed information on the visual characteristics that distinguish ADSD and MTD.

Subjects and Method 11 female patients of ADSD and 13 female patients of MTD who were diagnosed by laryngoscope and stroboscope from 2009 through 2012 were selected for this study. The speech samples of subjects were obtained using Computerized Speech Lab. The two speech therapists evaluated a wide-band $(300 \mathrm{~Hz})$ spectrogram by blind test using 4 scales (0-3 point) for four spectral findings.

Results Abrupt voice breaks and irregular wide-spaced vertical striations of ADSD were significantly higher than those of MTD. Well-defined formants and high-frequency spectral noise were not found significantly different between two groups.

Conclusion The spectrograms provided visual perceptual information needed to differentiate ADSD from MTD. Voice therapy to reduce hypertension could be considered for patients of ADSD with excessive formants and noise. If spectrogram analysis were used along with other assessments, it would be more useful in distinguishing ADSD from MTD.

Korean J Otorhinolaryngol-Head Neck Surg 2015;58(7):481-6

Key Words Adduct spasmodic dysphonia - Muscle tension dysphonia - Spectrogram . Voice.

\section{서 론}

내전형 연축성 발성장애(adduct spasmodic dysphonia, $\mathrm{ADSD})$ 는 끊김을 동반한 쥐어짜는 음성이 특징이며 신경학 적인 기능장애가 원인으로 알려져 있다. ${ }^{1)}$ 근긴장성 발성장애 (muscle tension dysphonia, MTD)는 그 증상이 ADSD와 유 사하나 후두근육의 과도한 긴장에 의한 기능적인 음성장애 로 분류된다. ${ }^{2,3)}$

두 질환은 유사한 증상에도 불구하고 치료방법이 상이하기 때문에 정확하고 다양한 진단방법들이 요구된다. ${ }^{4)}$ 두 질환을 
진단하는데 후두 스트로보스코피, 발성공기흐름측정(phonatory airflow measurement), 발성 끊김 분석(phonatory break analysis), 스펙트로그래피(spectrography), 발화과제에 따른 음향학적 분석, 청지각적 평가 등이 사용되며, 이러한 방법 들을 통하여 두 질환을 변별하고 또한 그 특징들이 연구되고 있다. ${ }^{5-9)}$ 그러나 주로 음성 끊김이 동반되지 않으면 ADSD가 아니라는 청지각적인 특성에 의존하여 이루어지고 있다.

두 질환의 특성을 살펴보는 방법 중 스펙트로그램 분석이 있다. Rees 등 ${ }^{8}$ 의 연구에서는 $\mathrm{ADSD}$ 와 MTD가 스펙트로그 램에서 몇 가지 차이를 보였으며, 그 특성으로 갑작스런 음성 의 끊김(abrupt voice breaks), 불규칙하고 넓은 수직적 간격 (irregular wide-spaced vertical striations), 선명한 포먼트 (well-defined formants), 고주파수 대역의 스펙트럼 잡음 (high-frequency spectral noise)에서 나타났다. 그리고 스펙 트로그램 분석은 청지각적 평가나 임상적 평가에서 두 질환 을 변별하기에 충분치 않은 경우에 중요한 정보를 제공해준 다고 하였다.

이에 저자들은 $\mathrm{ADSD}$ 와 $\mathrm{MTD}$ 를 변별할 때 스펙트로그램 분석이 시각적이고 세부적인 정보를 제공해줄 수 있는지에 대 해서 알아보고자 $\mathrm{ADSD}$ 와 MTD로 진단받은 환자들을 대상 으로 그 특성을 살펴보았다.

\section{대상 및 방법}

2008년 5월부터 2012년 12월까지 본원 이비인후과를 내원 하여 전반적인 음성평가를 통해 이비인후과 전문의와 음성언 어치료사에 의해 $\mathrm{ADSD}$ 와 MTD로 진단받은 총 24명을 대상 으로 하였다. 진단은 Cannito and Woodson의 기준에 의하였 으며, $\mathrm{ADSD}$ 는 청지각적 특징으로 음성일탈(phonatory break)

Table 1. Patients characteristics

\begin{tabular}{lccc}
\hline & ADSD & MTD & Total \\
\hline No. subjects & 11 & 13 & 24 \\
Average age (years) & 46.6 & 44.2 & 45.4 \\
Range (years) & $22-78$ & $22-70$ & $22-78$ \\
Sex & Female & Female & Female \\
Grade (0-3 point) & 1.91 & 2.08 & \\
\hline
\end{tabular}

ADSD: adduct spasmodic dysphonia, MTD: muscle tension dysphonia
과 쥐어짜는 음질(strained-strangled quality), 일시적으로 정 상적인 음성을 보이고, 비구어적인 발성과 고음에서 개선된 음질을 보이는 경우에 진단하였다. $\mathrm{MTD}$ 는 짧은 기간의 음 성치료로 음질의 개선을 보이고 과도한 후두긴장을 동반한 경우로 진단하였다. 대상자 중 $\mathrm{ADSD}$ 군은 11 명이며 평균나 이는 44.2세(22 70세), MTD군은 13명이며 평균나이는 46.6 세(22 78세)였고, 모두 여자였다(Table 1). 실험 전 청지각적 평가를 통한 두 집단 간의 전반적인 음질수준(grade: 0 3점) 은 $\mathrm{ADSD}$ 군은 1.91점, MTD군은 2.08점으로 평가되었다.

자료수집은 Computerized Speech Lab(Model 4150; Kay Electrics Corp., Lincoln Park, NY, USA)의 'CSL Main Program'을 이용하였고, 환자들에게 '가을' 문단의 첫 번째 문장 을 읽게 하여 광역대 $(300 \mathrm{~Hz})$ 스펙트로그램으로 분석하였다. 음성자료는 방음실에서 환자를 편안한 자세로 앉게 한 후, 다이나믹 마이크(shure SM48, SHURE, Niles, IL, USA)와 10 $\mathrm{cm}$ 거리를 두고 가능한 평상시와 같은 음도와 강도로 읽게 하였다. 분석은 이비인후과에서 음성검사 및 치료 경력이 있 는 2 명의 언어재활사(경력 3년 이상)가 blind test으로 평가하 였다. 스펙트로그램 평가는 4점 척도(0 3점)로 '갑작스런 음 성 끊김', '불규칙하고 넓은 수직적 간격, '고주파수 대역의 스 펙트럼 잡음 정도'는 '0점: clear, 1점: mild, 2점: moderate, 3 점: severe'로, '선명한 포먼트'는 '0점: absent, 1점: mild, 2점: well-defined, 3점: excessive'로 평가하였다. 평가자에게는 평가 전에 4가지 항목에 대한 정의와 기준에 대한 교육을 실 시하였고, 예시를 제공하여 실험 전 충분한 예비평가를 진행 하였다.

4가지 항목에 대한 정의는 다음과 같다(Table 2). '갑작스 런 음성 끊김은 문장 발화 시 유성음에서 발성의 끊김을 의 미한다. 여기서 음절과 음절 간의 stop과 구분하기 위하여 스 펙트로그램을 제시할 때 하단에 말소리를 표시하였다. '불규 칙하고 넓은 수직적 간격'에 대해 수직적 간격은 glottal pulsing을 의미하며 불규칙적인 간격은 쥐어짜는 음성과 관련이 있다. '선명한 포먼트'는 음향학적 에너지가 집중된 영역을 의 미한다. '고주파수 대역의 스펙트럼 잡음 정도'는 쉰 소리 (breathy voice)로 인하여 고주파수 대역에서 잡음이 형성된 것을 의미한다. ${ }^{10)}$

이러한 정의를 기준으로 4 가지 항목에 대해 스펙트로그램

Table 2. Definition of the spectral findings

\begin{tabular}{ll}
\hline Abrupt voice breaks & An absence of voicing that is not common to normally occurring phonetic segments \\
Irregular wide-spaced vertical striations & $\begin{array}{l}\text { The vertical striations represent glottal pulsing } \\
\text { Aperiodic wave type associated with strained-strangled phonation }\end{array}$ \\
Well-defined formants & $\begin{array}{l}\text { Clear resonant frequencies } \\
\text { Major areas of concentration of acoustic energy }\end{array}$ \\
High-frequency spectral noise & Noise in high frequency associated with breathy voice quality \\
\hline
\end{tabular}




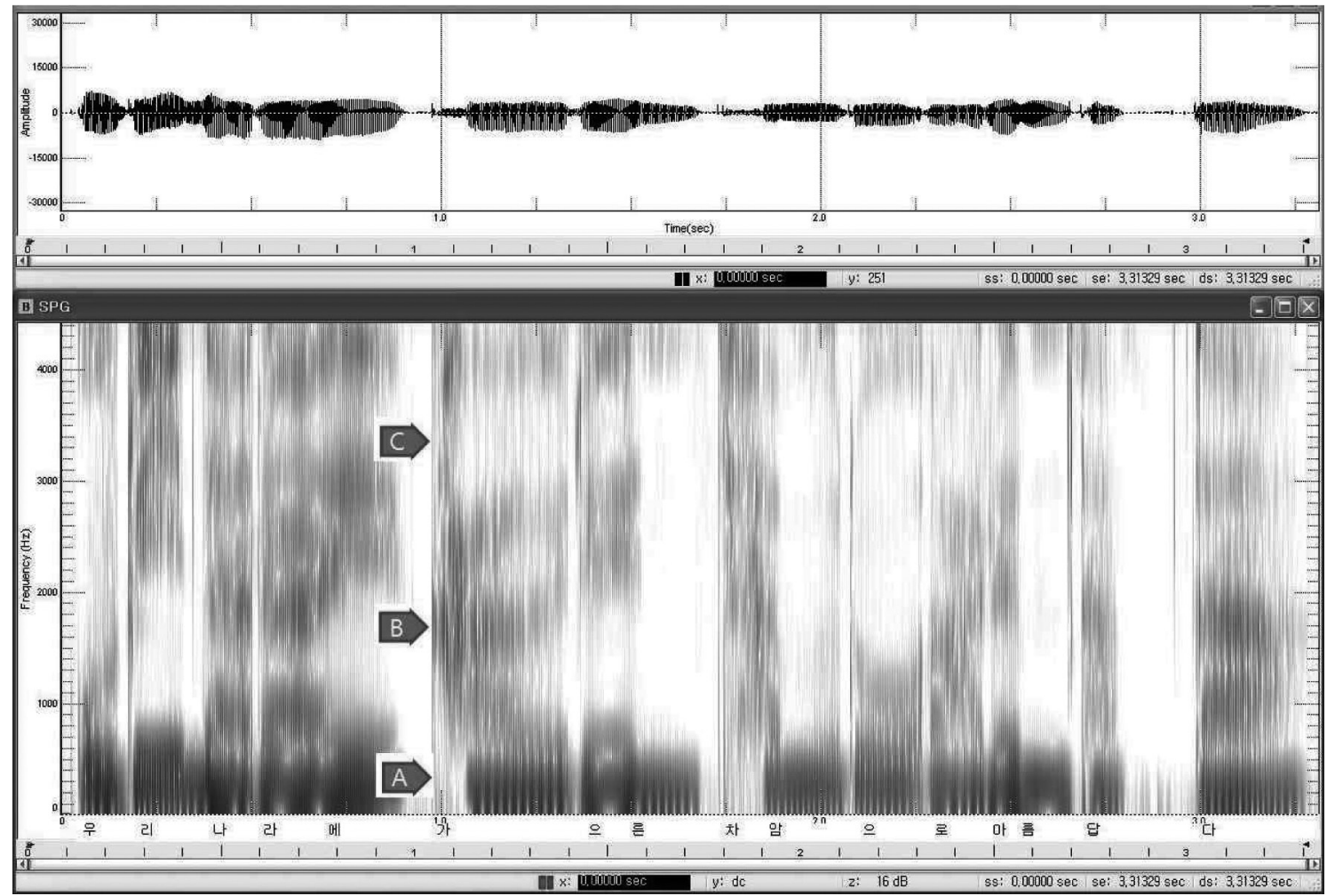

Fig. 1. Representative spectrogram from a normal speaker. Regularly wide-spaced vertical striations (A), well-defined formants (B) and little high-frequency spectral noise (C) during speech (arrows).

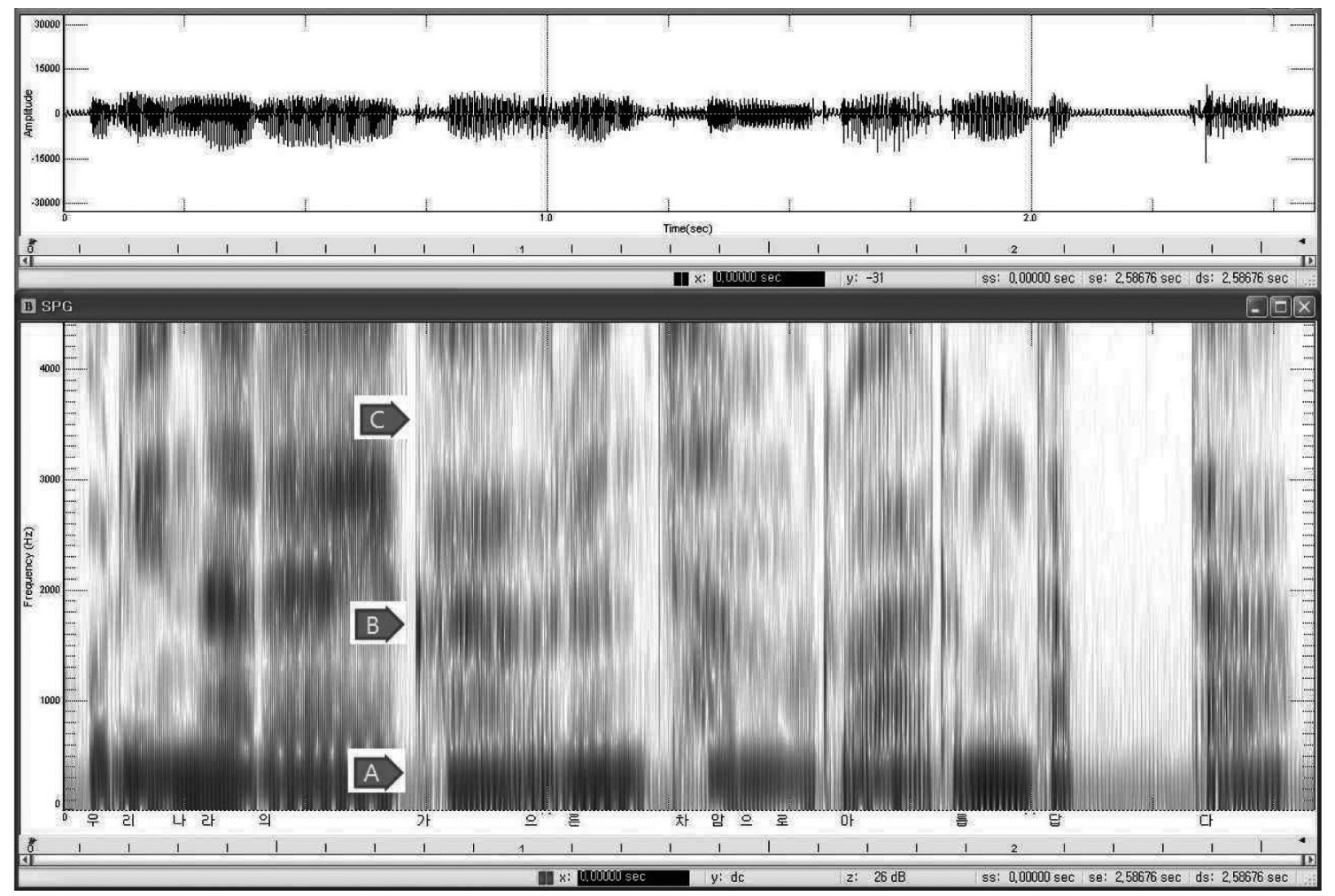

Fig. 2. Representative spectrogram from a MTD subject. No abrupt voice break, but irregular wide-spaced vertical striations (A: 2 pointmoderate), well-defined formants (B: 2 point-moderate) and high-frequency spectral noise (C: 2 point-moderate) during speech (arrows). MTD: muscle tension dysphonia. 


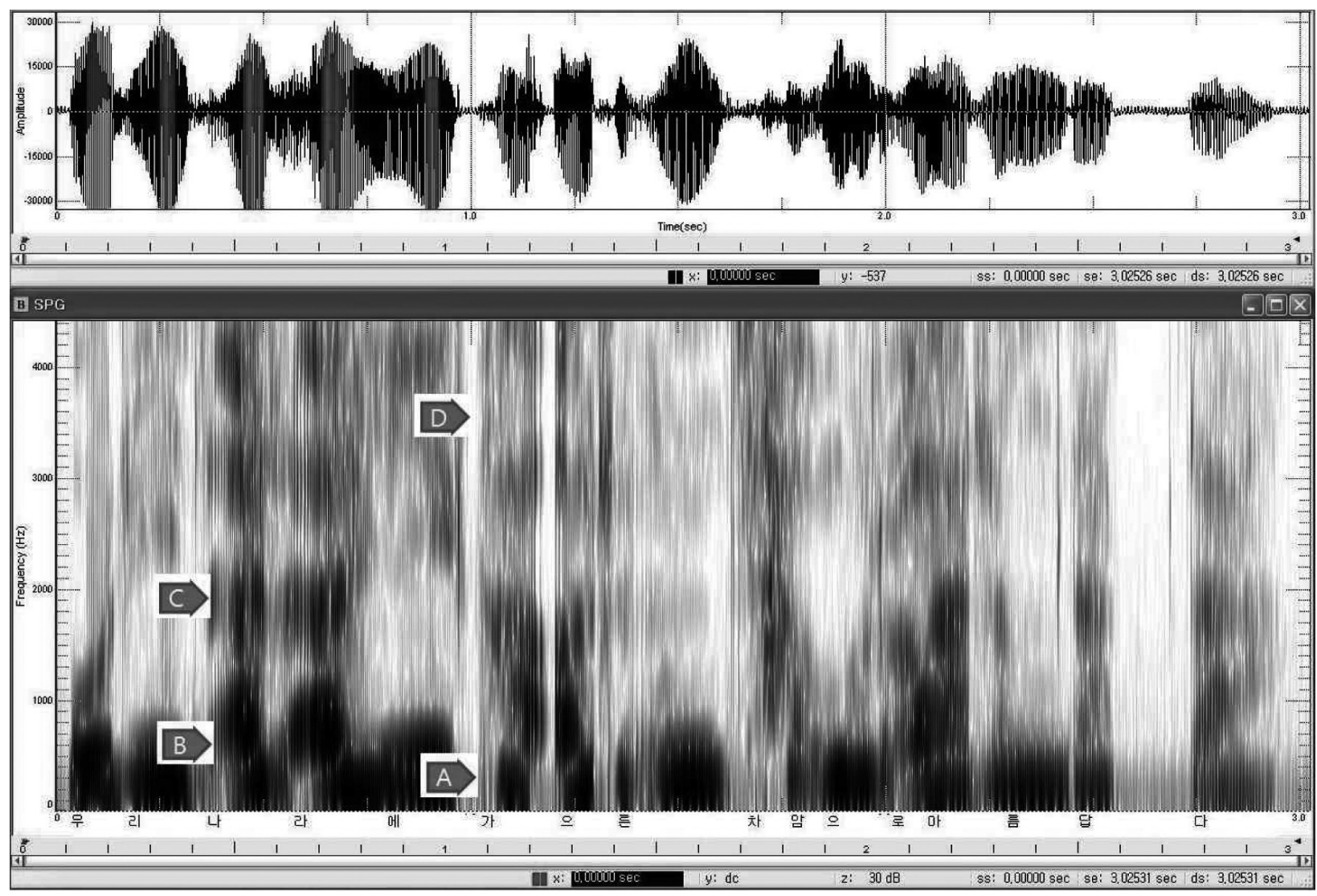

Fig. 3. Representative spectrogram from an ADSD subject. Abrupt voice break (A: 2 point-moderate), irregular wide-spaced vertical striations (B: 2 point-moderate), formants (C: 3 point-excessive), and high-frequency spectral noise (D: 3 point-severe). ADSD: adduct spasmodic dysphonia.

을 통해 평가를 실시하였다(Figs. 1, 2, and 3).

수집된 자료는 통계분석 프로그램 Statistical Package for the Social Sciences(SPSS, version 12.0, Chicago, IL, USA) 로 분석하였다. 각 항목에 대하여 두 집단 간의 차이는 독립 표본 t-검증(independent sample t-test)을 실시하였고, 통계 검정상 $p$-value $<0.05$ 를 유의한 것으로 간주하였다. 평가자 간-내 신뢰도를 측정하기 위해 피어슨 상관계수(Pearson r correlation)로 분석하였다.

\section{결 과}

\section{평가자 간-내 신뢰도}

평가자 간 신뢰도는 $\mathrm{r}=0.738 \sim 0.824(p<0.001)$ 로 상관이 높 은 것으로 나타났으며, 평가자 내 신뢰도는 $\mathrm{r}=0.032 \sim 0.972$ $(p<0.001)$ 로 상관이 매우 높은 것으로 나타났다(Table 3).

\section{4가지 항목에 대한 스펙트로그램 특성 비교}

'갑작스런 음성 끊김'과 ‘불규칙하게 넓은 수직적 간격'에 대해 두 집단 간 유의한 차이를 보였다. 하지만 선명한 포먼
Table 3. Inter and intra-rater reliability

\begin{tabular}{lcc}
\hline & Inter-rater & Intra-rater \\
\hline Abrupt voice breaks & $0.824^{*}$ & $0.972^{*}$ \\
$\begin{array}{l}\text { Irregular wide-spaced vertical } \\
\text { striations }\end{array}$ & $0.814^{*}$ & $0.827^{*}$ \\
Well-defined formants & $0.738^{*}$ & $0.923^{*}$ \\
High-frequency spectral noise & $0.746^{*}$ & $0.832^{*}$ \\
\hline
\end{tabular}

Pearson $r$ correlation coefficient measures show high interrater and intrarater reliability in all findngs. $* p<0.001$

트'와 '고주파수 대역에서의 잡음 정도'에서는 유의한 차이가 없었다 $(p<0.05)$. '갑작스런 음성 끊김'에 대한 $\mathrm{ADSD}$ 군의 평 균점수는 $1.455 \pm 0.934$ 점, $\mathrm{MTD}$ 군은 $0.615 \pm 0.650$ 점으로 두 집단 간 통계적으로 유의한 차이가 있었고, $\mathrm{ADSD}$ 군에서 높 은 결과를 보였다 $(p=0.022)$. '불규칙하게 넓은 수직적 간격'에 대한 $\mathrm{ADSD}$ 군의 평균점수는 $2.273 \pm 0.647$ 점, $\mathrm{MTD}$ 군은 $1.462 \pm 0.519$ 점으로 두 집단 간 통계적으로 유의한 차이가 있었고, $\mathrm{ADSD}$ 군에서 더 높은 결과를 보였다 $(p=0.003)$. '선 명한 포먼트'에 대해서는 $\mathrm{ADSD}$ 군의 평균점수는 $1.727 \pm$ 0.647점, $\mathrm{MTD}$ 군은 $1.692 \pm 0.480$ 점으로 두 집단 간 통계적으 로 유의한 차이를 보이지 않았다. 그러나 $\mathrm{ADSD}$ 군이 MTD군 
Table 4. Spectral findings in ADSD and MTD

\begin{tabular}{lccc}
\hline & ADSD & MTD & $p$-value \\
\hline Abrupt voice breaks & $1.455(0.934)$ & $0.615(0.650)$ & $0.022^{*}$ \\
Irregular wide-spaced vertical striations & $2.273(0.647)$ & $1.462(0.519)$ & $0.003^{*}$ \\
Well-defined formants & $1.727(0.647)$ & $1.692(0.480)$ & 0.926 \\
High-frequency spectral noise & $1.545(0.522)$ & $1.769(0.599)$ & 0.339 \\
\hline
\end{tabular}

Data are given as mean (standard deviation). There are significant correlations between ADSD and MTD in abrupt voice breaks and irregular wide-spaced vertical striations. $* p<0.05$. ADSD: adduct spasmodic dysphonia, MTD: muscle tension dysphonia

에 비해 더 높은 결과를 보였다 $(p=0.926)$. '고주파수 대역에 서의 잡음 정도'에서 $\mathrm{ADSD}$ 군의 평균점수는 $1.545 \pm 0.522$ 점, $\mathrm{MTD}$ 군은 $1.769 \pm 0.599$ 점으로 두 집단 간 통계적으로 유의 한 차이는 보이지 않았으나 $\mathrm{MTD}$ 군이 $\mathrm{ADSD}$ 군에 비해 더 높은 결과를 보였다 $(p=0.339)$ (Table 4).

\section{고 찰}

스펙트로그램은 주파수와 진폭(강도)의 시간에 따른 변화 를 보여주는 삼차원적인 그림이며, 광역대 스펙트로그램은 포먼트라 불리는 공명주파수의 에너지 영역뿐 아니라 음성 의 주기성을 관찰할 수 있다.11) 스펙트로그램 분석을 통하여 $\mathrm{ADSD}$ 와 $\mathrm{MTD}$ 의 특성을 살펴볼 수 있으며, '갑작스런 음성 의 끊김' '불규칙하고 넓은 수직적 간격', '선명한 포먼트', '고 주파수 대역의 스펙트럼 잡음 정도'를 기준으로 두 질환을 구 분할 수 있다.

본 연구에서 두 집단 모두 '갑작스런 음성 끊김'을 관찰할 수 있었고, $\mathrm{ADSD}$ 군이 $\mathrm{MTD}$ 군에 비해 높은 결과를 보였으 며 통계적으로도 유의한 차이가 있었다. 따라서 두 질환을 구분할 때 주요한 기준이 되는 특징이 음성 끊김(phonatory breaks)이며, 이를 스펙트로그램 상에서도 시각적으로 확인 할 수 있었다. Rees 등 ${ }^{8}$ 은 음성 끊김이 $\mathrm{ADSD}$ 군에서만 관찰 되었고, $\mathrm{MTD}$ 군에서는 관찰되지 않았다고 보고한 바 있다. 그러나 Roy 등 ${ }^{12)}$ 은 $\mathrm{ADSD}$ 보다는 적지만 $\mathrm{MTD}$ 에서도 문장 산출 동안 음성 끊김을 보였다고 하였고, Sapienza 등 ${ }^{13)}$ 도 여 자 MTD 환자들의 거의 $50 \%$ 에서 적어도 한 번 이상의 음성 끊김이 관찰되었다고 보고하였다. 본 연구 또한 문장산출 동 안의 두 집단의 음성특성을 분석하였으며, 이들과 같이 MTD 군에서도 음성 끊김이 관찰되었으나, 정도는 $\mathrm{ADSD}$ 군에 비 해 낮은 결과를 보였다. 또한 일부 연구에서는 두 질환을 진 단할 때 다양한 발화과제상황에서 살펴보기도 하기도 하는 데, $\mathrm{ADSD}$ 는 '과제의존도(task-specific)'가 높아 모음연장발 성에서 음성 끊김이 관찰되지 않을 수 있지만 문장산출 동안 에 끊김을 보인다고 하였고, 유성음과 무성음에 따른 변화가 큰 반면, MTD는 과제에 따른 특성 차이가 거의 없다고 하였 다. ${ }^{7,914)}$ 따라서 두 질환을 구분할 때 다양한 발화과제를 통한
진단도 요구된다.

'불규칙하고 넓은 수직적 간격'은 $\mathrm{ADSD}$ 군이 $\mathrm{MTD}$ 군에 비 해 더 높은 결과를 보였고, 통계적으로도 유의한 차이를 보였 다. Rees 등 ${ }^{8}$ 의 연구에서도 $\mathrm{ADSD}$ 와 $\mathrm{MTD}$ 군 모두 이러한 특징이 보인다고 하였는데, 그 정도는 $\mathrm{ADSD}$ 가 높았다. 스펙 트로그램에서 규칙적인 수직적 간격은 음성신호의 준주기적 인 파형유형(quasi-periodic wave type)을 의미하며, 불규칙 하고 넓은 수직적 간격은 내전근 경련(adductor spasm)으로 인한 쥐어짜고 조이는 발성(strained-strangled phonation) 과 관련 지을 수 있다. 따라서 청지각적으로 힘을 주고 조이는 발성양상이 스펙트로그램에서는 수직적 간격이 넓고 비주기 적인 형태로 관찰된다는 것을 알 수 있다.

'포먼트 형성 정도'를 분석한 결과, 두 집단 간 통계적으로 유의한 차이는 없었으나, $\mathrm{ADSD}$ 군이 $\mathrm{MTD}$ 군에 비해 높은 결 과를 보였다. 포먼트(formant)는 공명 강(resonant cavities) 의 크기나 형태의 변화에 의해 에너지의 주파수가 형성되는 영역이며, 정상음성에서는 선명하고 뚜렷하다. 그러나 불완전 한 성대내전은 쉰 음성(breathy voice quality)과 함께 스펙 트로그램상에서는 공명에너지 형성을 어렵게 한다. ${ }^{10)}$ Rees 등 $^{8)}$ 의 연구에서는 $\mathrm{ADSD}$ 에서 포먼트 형성이 잘 이루어졌으 며, MTD에서는 관찰이 되지 않았다고 하였다. 본 연구에서 도 $\mathrm{ADSD}$ 군에서 포먼트가 잘 발달된 것을 확인하였다. 그러 나 Rees 등 $^{8}$ 의 연구와 달리 MTD군에서도 비교적 포먼트 형 성이 잘 이루어진 것이 관찰되었다. 이러한 차이는 대상자 선 정에 따른 차이인 것으로 보이는데, 본 연구에서는 다른 질 환이 동반되지 않은 MTD만을 대상으로 하였고, 반면 Rees 등 $^{8)}$ 의 연구에서는 성대마비와 같은 불완전한 성대내전으로 인한 MTD였기 때문에 포먼트 형성에 어려움을 보인 것으로 보인다. 또한 본 연구의 $\mathrm{ADSD}$ 군에서 과도하게 포먼트가 형 성된 환자들이 있었는데, 이러한 특징은 과도하게 힘을 주고 발성한 결과로 스펙트로그램을 통해 과도한 긴장과 쥐어짜 는 발성패턴에 대한 것을 관찰할 수 있었다.

마지막으로 스펙트로그램을 통해 살펴본 항목 중 '고주파 수 대역에서의 잡음은 두 집단 간 통계적 유의성이 없었다. 그 러나 $\mathrm{ADSD}$ 군에 비해 MTD군에서 높은 점수를 보였다. 성대 내전의 어려움으로 인한 과도한 쉰소리는 스펙트로그램상 공 
명의 붕괴(breakdown)와 더불어 고주파수 대역에서 잡음을 만들어낸다. ${ }^{10)}$ Rees 등 ${ }^{8}$ 의 연구에서 $\mathrm{MTD}$ 군은 과도한 고주 파수 대역에서 잡음이 관찰되었고, 반면 $\mathrm{ADSD}$ 군에서는 일 부에서만 관찰되었다. 신경학적이나 기질적인 문제가 동반되 지 않지만 과도한 후두 내외근육의 긴장으로 인해 성대진동 이 감소해 MTD 환자에서도 쉰소리가 발생한다. ${ }^{15)}$ 이러한 특 징은 $\mathrm{ADSD}$ 군의 특징과 차이를 보이며 스펙트로그램 분석 을 통해 시각적으로 확인할 수 있었다.

\section{REFERENCES}

1) Cannito MP, Woodson G. The spasmodic dysphonias. In: Kent RD, Ball MJ, editors. Voice quality measurement. San Diego: Singular Thomson Learning;2000. p.411-30.

2) Ludlow CL. Treatment for spasmodic dysphonia: limitations of current approaches. Curr Opin Otolaryngol Head Neck Surg 2009; 17(3):160-5.

3) Morrison MD, Rammage LA. Muscle misuse voice disorders: description and classification. Acta Otolaryngol 1993;113(3):428-34.

4) Boone DR, McFarlane SC, VonBerg SL. The voice and voice therapy. 7th ed. Boston: Allyn \& Bacon;2004. p.114-21.

5) Leonard R, Kendall K. Differentiation of spasmodic and psychogenic dysphonias with phonoscopic evaluation. Laryngoscope 1999;109(2 Pt 1):295-300.

6) Higgins MB, Chait DH, Schulte L. Phonatory air flow characteristics of adductor spasmodic dysphonia and muscle tension dysphonia. J Speech Lang Hear Res 1999;42(1):101-11.
7) Roy N, Mauszycki SC, Merrill RM, Gouse M, Smith ME. Toward improved differential diagnosis of adductor spasmodic dysphonia and muscle tension dysphonia. Folia Phoniatr Logop 2007;59(2):8390.

8) Rees CJ, Blalock PD, Kemp SE, Halum SL, Koufman JA. Differentiation of adductor-type spasmodic dysphonia from muscle tension dysphonia by spectral analysis. Otolaryngol Head Neck Surg 2007;137(4):576-81.

9) Roy N, Gouse M, Mauszycki SC, Merrill RM, Smith ME. Task specificity in adductor spasmodic dysphonia versus muscle tension dysphonia. Laryngoscope 2005;115(2):311-6.

10) Rontal E, Rontal M, Rolnick MI. The use of spectrograms in the evaluation of vocal cord injection. Laryngoscope 1975;85(1):47-56.

11) Kent RD, Read C. The acoustic analysis of speech. 2nd ed. San Diego: Singular/Thomson Learning;2002. p.57-61.

12) Roy N, Whitchurch M, Merrill RM, Houtz D, Smith ME. Differential diagnosis of adductor spasmodic dysphonia and muscle tension dysphonia using phonatory break analysis. Laryngoscope 2008;118 (12):2245-53.

13) Sapienza CM, Walton S, Murry T. Adductor spasmodic dysphonia and muscular tension dysphonia: acoustic analysis of sustained phonation and reading. J Voice 2000;14(4):502-20.

14) Erickson ML. Effects of voicing and syntactic complexity on sign expression in adductor spasmodic dysphonia. Am J Speech Lang Pathol 2003;12(4):416-24.

15) Patel RR, Liu L, Galatsanos N, Bless DM. Differential vibratory characteristics of adductor spasmodic dysphonia and muscle tension dysphonia on high-speed digital imaging. Ann Otol Rhinol Laryngol 2011;120(1):21-32. 